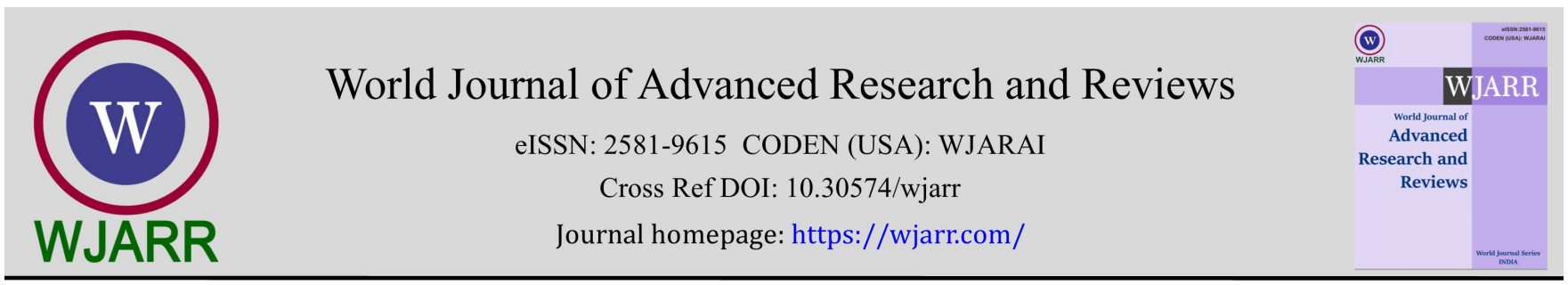

(RESEARCH ARTICLE)

Check for updates

\title{
Effect of proprioceptive sensation and feedback required for motor learning on function, activity, and daily life behavior of patients after nerve injury
}

Jung-Ho Lee *

Department of Physical Therapy, Kyungdong University: 815, Gyeonhwon-ro, Munmak-eup, Wonju-si, Gangwon-do, Republic of Korea.

World Journal of Advanced Research and Reviews, 2021, 11(03), 474-481

Publication history: Received on 12 August 2021; revised on 18 September 2021; accepted on 20 September 2021

Article DOI: https://doi.org/10.30574/wjarr.2021.11.3.0462

\begin{abstract}
Purpose: This study investigated the effects of modified mirror therapy and taping therapy for providing feedback on upper extremity function, activity, and daily activities of stroke patients.

Methods: This study was conducted with 20 stroke patients and randomly assigned 10 subjects to the experimental group and 10 to the control group. In the experimental group, after proprioceptive neuromuscular stimulation treatment, the mirror therapy program was implemented. In the control group, upper extremity taping was performed before proprioceptive neuromuscular facilitation treatment. In this study, a prior evaluation using JTT, FIM, and MAL was performed before intervention to evaluate the patient's function and daily life behavior. Post-tests were performed after the last treatment.
\end{abstract}

Results: In the paired-sample T-test used for within-group comparison of JTTs, FIM, and MAL, there was a significant difference between pre- and post-test for all groups. But there was no statistically significant difference between experimental group 1 and experimental group 2 in an independent t-test conducted to compare the effect sizes of treatments.

Conclusion: In other words, by applying mirror therapy and taping therapy that can increase proprioceptive sensation and feedback information in stroke patients, upper extremity function and daily activities can be increased.

Keywords: Mirror therapy; Taping; Rehabilitation; Function; Stroke

\section{Introduction}

Proprioception is used to predict various injuries because it significantly correlates with the ability to maintain postures. It is the ability to form joint angles with the positional senses, which provide information about the body's position, the angles and angular velocities of all joints involved in movements on individual movement planes, and the ratios of their movements to the central nervous system [1].

Moreover, proprioception transmits information from pulled and stretched muscles or ligaments to the brain in order to determine how much force should be applied, and it is essential in accurately performing complex movements. Proprioception plays the most significant role in maintaining dynamic joint stability, and it also induces normal movements and protects the joints from external damage [2].

\footnotetext{
${ }^{*}$ Corresponding author: Jung-Ho Lee

Department of Physical Therapy, Kyungdong University: 815, Gyeonhwon-ro, Munmak-eup, Wonju-si, Gangwon-do, Republic of Korea.
}

Copyright (c) 2021 Author(s) retain the copyright of this article. This article is published under the terms of the Creative Commons Attribution Liscense 4.0. 
Additionally, various types of senses affect the movement senses and joint positions, including sight and skin sensations. If the positional senses are lost, motor abilities degrade, and difficulties occur in daily living activities. Proprioception is a conscious sense of joint movements and positions, and it has been used as an important factor in the prognoses of rehabilitation patients [3].

From a neurophysiological perspective, the central nervous system plays a role in generating motor commands and body movements through processing the sensory information transmitted from the peripheral nervous system. Additionally, when a complex movement or new movement is performed, this process is more complex and organic, and movements can be performed accurately only when information processing is smoothly carried out [4].

Generally, proprioception results in a loss of the positional senses and secondary diseases after severe tissue damage or chronic pain. Thus, proprioception changes occur alongside changes to the sensitivity of the muscle spindle nerve fibers, which relay the positional senses during movement, as well as changes to information transmission abilities to the central nervous system. Thus far, studies on various kinds of stimulation training to improve proprioception have been conducted in order to prevent damage, such as falls, among patients experiencing hypoesthesia due to disease and among elderly patients [5].

Movement disorders caused by damage to the central nervous system, such as stroke, make it difficult to move normally, and patients often use compensatory action to solve this problem. Characteristics of disability that occur mainly after stroke are stiffness, muscle weakness, and hypertonia, which affect the proper movement control of the upper extremities, and reduce the range of motion of the joints, which limits independent daily life performance. As a result, it becomes difficult to use the normal upper extremity movement of the injured side, and in order to solve this problem, the non-injured side is used inefficiently to induce abnormal movements [6].

Therefore, this study was conducted to investigate the effects of mirror therapy and taping therapy, which can provide correct feedback and proprioceptive sensation, on upper extremity function and daily activities of stroke patients.

\section{Material and methods}

\subsection{Subjects}

This study was conducted with 20 stroke patients and randomly assigned 10 subjects to the experimental group and 10 to the control group (Figure 1). In the experimental group, after proprioceptive neuromuscular stimulation treatment, the mirror therapy program was implemented for 30 minutes, 3 times a week, a total of 12 times.

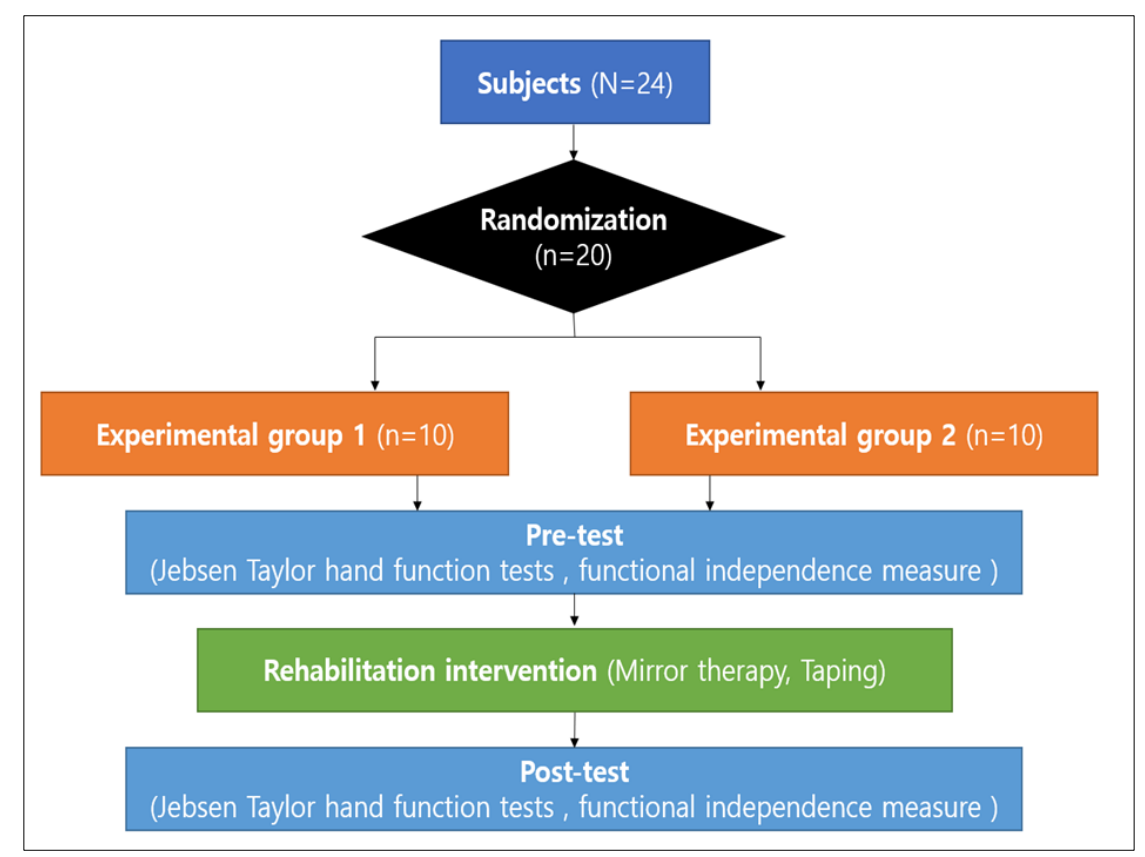

Figure 1 Flow chart 
In the control group, upper extremity taping was performed before proprioceptive neuromuscular facilitation treatment, which was performed 3 times a week for a total of 12 times.

Prior to the intervention, the function and ability of the paralyzed upper extremity and activities of daily living were pre-evaluated, and a post-test was conducted at 4 weeks after the intervention was completed. All evaluations were performed by the same physical therapist with more than 10 years of clinical experience to maintain consistency.

\subsection{Study design}

All study subjects heard a detailed explanation of the contents and process of the experiment before the experiment, and after signing the handwritten consent form to participate in the study, they participated in the study. All experimental procedures were carried out based on the Declaration of Helsinki.

\subsection{Inclusion criteria}

Inclusion criteria were set as those

- Subjects had consented to the treatment program

- Subjects who Brunnstrom's recovery had been at least at stage 4

- Subjects who received at least 24 points or more points on the mini-mental state examination

- Subjects without cognitive impairment

- $\quad$ Subjects had no problems with vision or hearing

\section{Evaluation method}

\subsection{Jebsen Taylor hand function tests}

Jebsen Taylor hand function tests (JTTs) were conducted to evaluate all participants' hand functions. The Jebsen Taylor hand function tests hand function activities frequently used in daily life, and it is widely used in clinical practice because it enables fast evaluations. All participants were asked to sit opposite the tester in a brightly lit room, and the heights of the desk and chair used for this test were adjusted so that each patient felt comfortable. The test began when a participant was comfortable and after each task had been sufficiently explained to the participant.

Using a standardized stopwatch, measurements were obtained for six of the test's seven items-excepting the writing of short sentences. Thus, the items included card flipping, picking up small objects and putting them into a can, pretending to eat, stacking chips, moving large and light cans, and moving large and heavy cans. The time taken to carry out each item was measured. Five sessions of training were administered for each item, and the average value of the three measurements was used as each participant's Jebsen Taylor hand function tests value.

\subsection{Functional independence measure}

Functional independence measure (FIM) is a tool for evaluating activities of daily living and is widely used to objectively evaluate the ability of patients with disabilities to perform activities of daily living. In this study, functional independence measure was used to evaluate the performance of daily living activities of stroke patients. It is an evaluation tool that is sensitive to functional changes as a method to objectively evaluate the life ability of patients with disabilities.

Compared to other evaluation tools, the functional independence measure is characterized by including evaluation of social cognition, and depending on the degree of help for each item, it is scored according to the degree of help for each item on a 7-point scale from complete dependence to independence, and the total score is given. is 126 points.

\subsection{Motor activity log}

Motor activity log (MAL) is a tool that examines the amount of use of the paralyzed upper extremity and the quality of movement of the paralyzed upper extremity in real daily life for hemiplegic patients after stroke through an interview. It is classified into Amount of Use on the paralyzed side and Quality of Movement for how much quality it is used.

The AOU score ranges from 0 (not used at all) to 5 (use the injured side as much as before the onset), and the QOM score ranges from 0 (the injured side cannot be used for the activity) to 5 (the injured side is used as before the onset). 
possible) on a 6-point scale. The higher the score, the better the amount of use and the quality of movement on the paralyzed side.

\section{Treatment methods}

\subsection{Mirror therapy}

For mirror treatment in this study, the experimental group was performed in a sitting position on a chair, and the mirror was erected vertically away from the patient in the sagittal plane on the desk. In this study, in order to apply the modified mirror therapy, both the paralyzed and non-paralyzed sides were projected onto the mirror using a double-sided mirror.

The patient's neutral line was maintained by placing the non-paraplegic and paralyzed hands between the mirrors. When the first program started, the patient was asked to gaze at the upper extremity of the non-paraplegic side reflected in the mirror. After that, they were asked to stare at the upper extremity of the paralyzed side while performing the same movement. Forearm pronation, supination, wrist and finger flexion, and extension were sequentially alternated on the paralyzed and non-paralyzed sides.

In addition, feedback was given to the patient as the therapist verbally explained the error in the movement.

\subsection{Taping therapy}

In this study, elastic taping was used to apply taping treatment. In this study, taping was applied to the shoulder region in order to apply taping therapy to the upper limb. For shoulder taping, elastic taping was applied to the deltoid, biceps brachii and supraspinatus muscles. For deltoid taping, elastic tape was attached from the rough surface of the humerus, the front part was attached to the coronoid process in a state of backward extension and slight abduction, and the rear part was attached to the scapula after placing the hand on the opposite.

For the biceps taping, the lower part of the (Y) shape was fixed to the glenoid fossa with the arm abducted and externally rotated, and then spread apart as if wrapped around the biceps with two pieces of tape. The supraspinatus taping was applied in the direction of the supraspinatus of the scapula at the great node of the affected humerus. Patients were instructed to remove all tapings within 48 hours of application.

\section{Statistical analysis method}

For analysis of the collected data, the mean and standard deviation of the dependent variable were obtained using the SPSS version 18.0 statistical processing program. An independent sample T-test was used to examine the homogeneity of upper extremity function and daily living activity performance on the paralyzed side between experimental group 1 and experimental group 2 before treatment intervention.

A paired-sample T-test was performed for the before and after comparison of upper extremity function and daily living activity performance within the groups of experimental group 1 and experimental group 2. In addition, an independent T-test was performed to compare the treatment effects between groups on upper extremity function and daily living performance. The statistical significance level $\alpha$ was set to 0.05 .

\section{Results}

In the results of the Jebsen Taylor hand function tests in this study, there was a statistically significant difference in the pre-evaluation and the post-evaluation between the experimental group 1 and the experimental group 2 in the pairedsample T-test $(P<0.05)$ (Figure 2). There was no statistically significant difference between experimental group 1 and experimental group 2 in an independent t-test conducted to compare the effect sizes of treatments $(P>0.05)$.

In the paired-sample T-test used for within-group comparison of functional independence measure scores, there was a significant difference between pre- and post-test for all groups $(P<0.05)$ (Figure 3$)$. There was no statistically significant difference between experimental group 1 and experimental group 2 in an independent t-test conducted to compare the effect sizes of treatments $(P>0.05)$. 


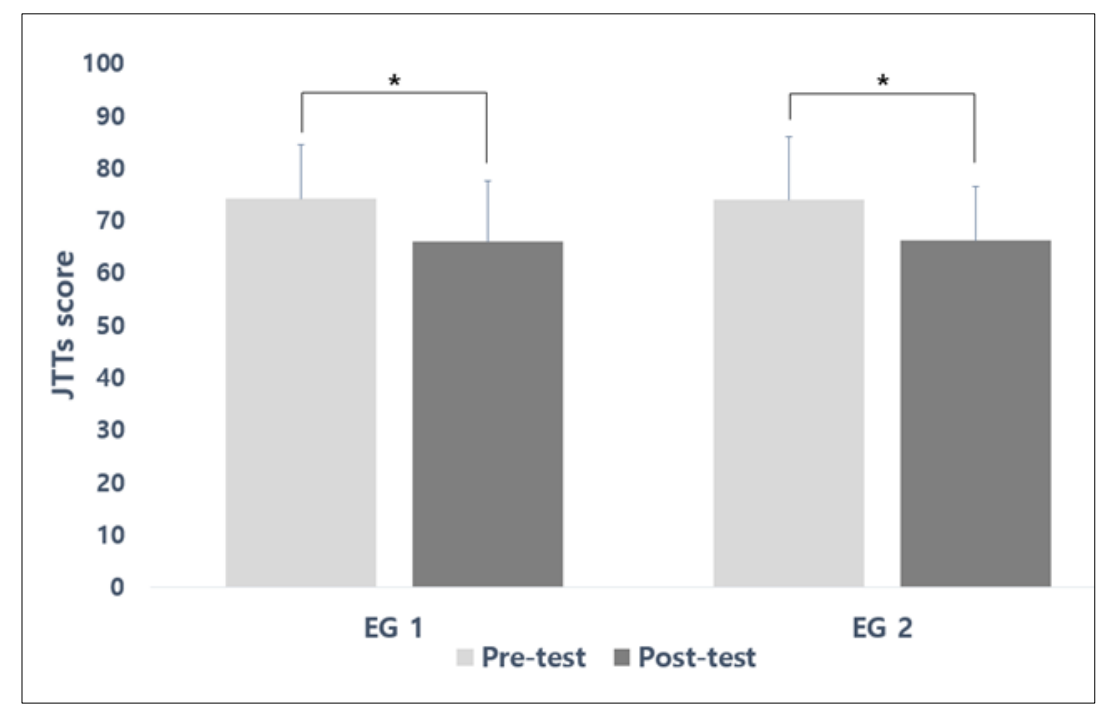

Figure 2 Comparison of Jebsen Taylor hand function between groups ${ }^{*} P<0.05$, EG 1: Experimental group 1, EG 2: Experimental group 2

In the paired-sample T-test used for within-group comparison of functional independence measure scores, there was a significant difference between pre- and post-test for all groups $(P<0.05)$ (Figure 3). There was no statistically significant difference between experimental group 1 and experimental group 2 in an independent $t$-test conducted to compare the effect sizes of treatments $(P>0.05)$.

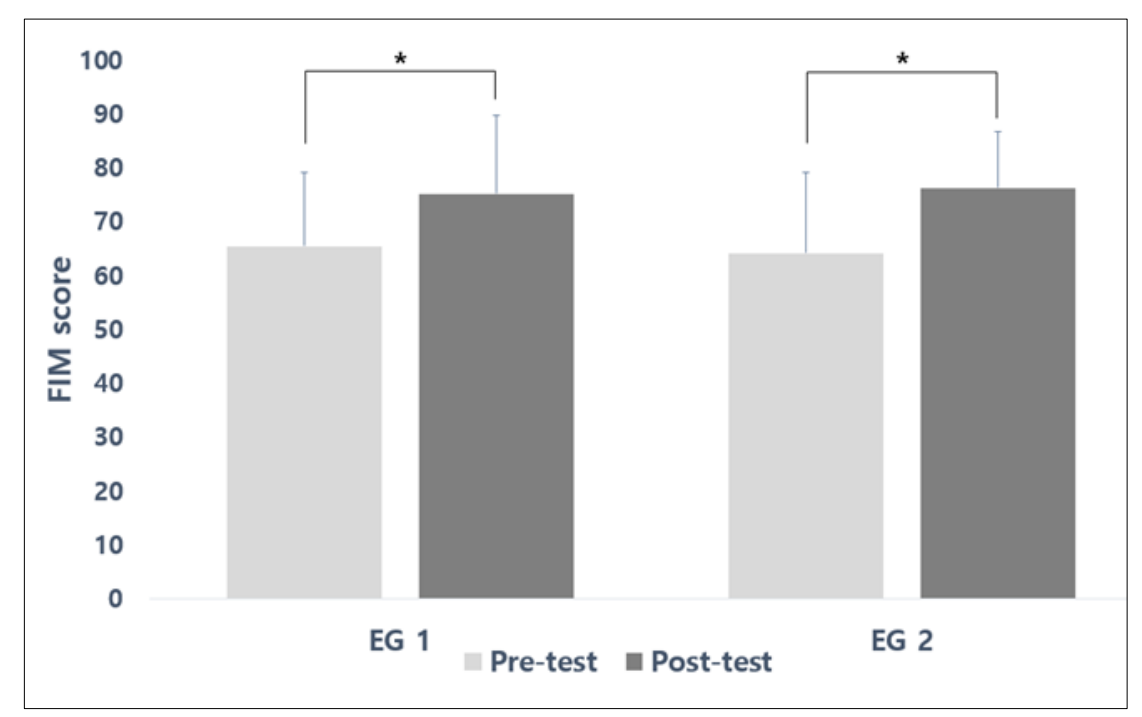

Figure 3 Comparison of functional independence measure between groups ${ }^{*} P<0.05$, EG 1: Experimental group 1, EG 2: Experimental group 2

In the paired-sample T-test used for within-group comparison of AOU scores of motor activity log, there was a significant difference between pre- and post-test for all groups $(P<0.05)$ (Figure 4). There was no statistically significant difference between experimental group 1 and experimental group 2 in an independent t-test conducted to compare the effect sizes of treatments $(P>0.05)$. 


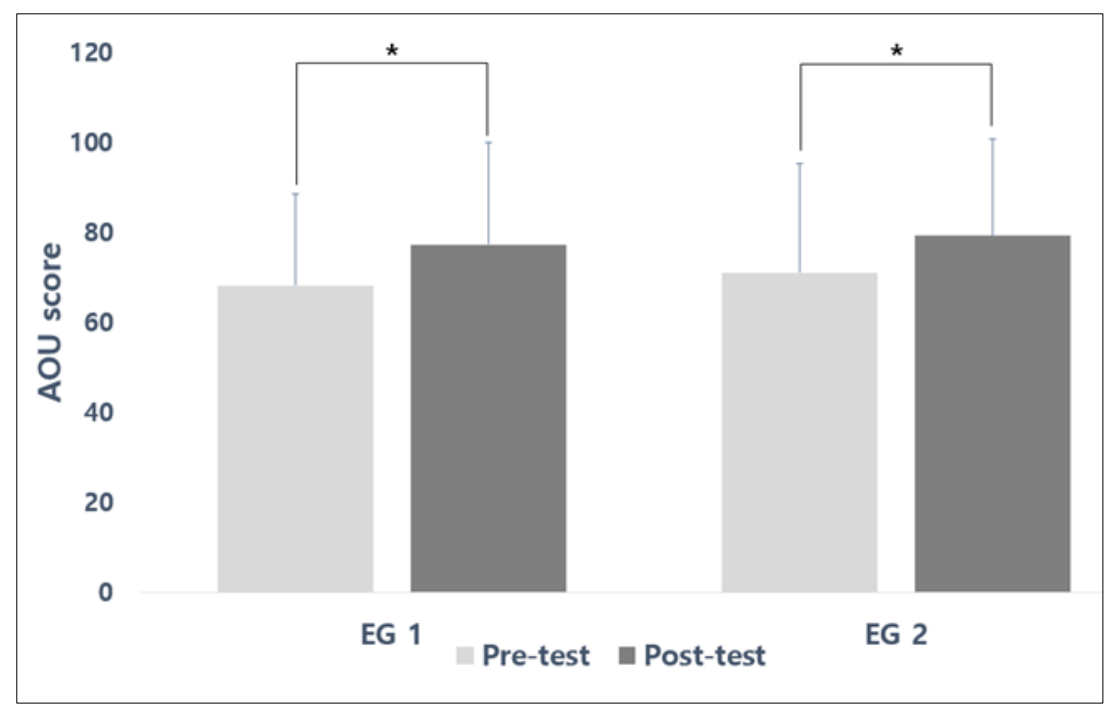

Figure 4 Comparison of AOU of motor activity log between groups ${ }^{*} P<0.05$, EG 1: Experimental group 1, EG 2: Experimental group 2, AOU: Amount of use

In the paired-sample T-test used for within-group comparison of QOM scores of motor activity log, there was a significant difference between pre- and post-test for all groups $(P<0.05)$ (Figure 4). There was no statistically significant difference between experimental group 1 and experimental group 2 in an independent $t$-test conducted to compare the effect sizes of treatments $(P>0.05)$.

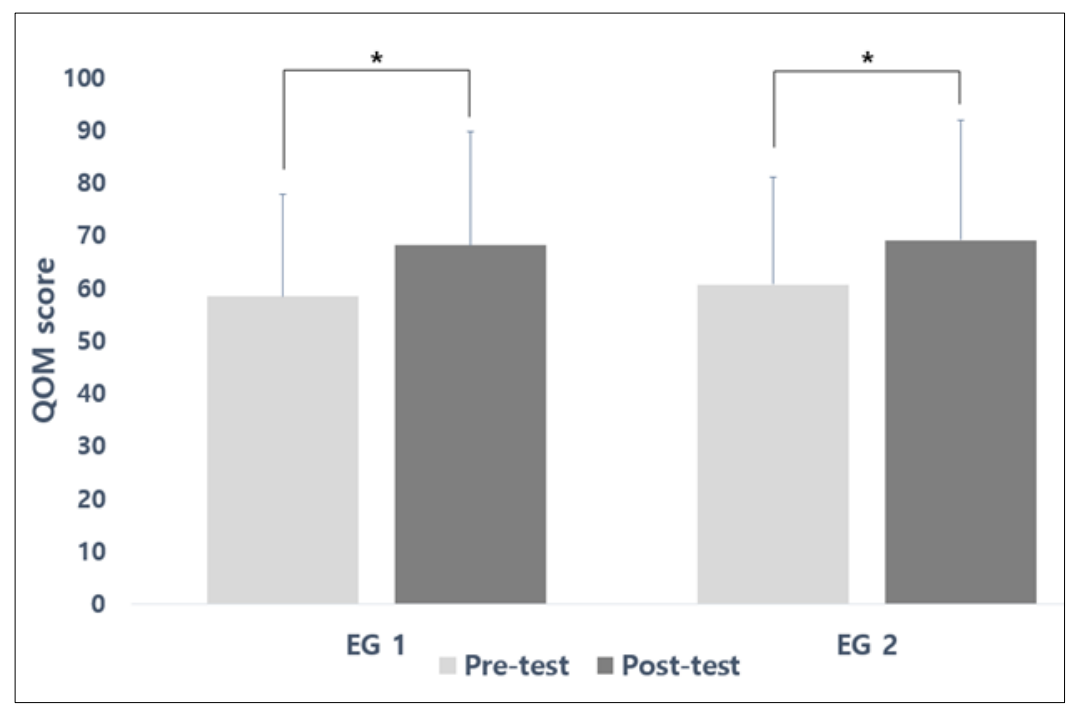

Figure 5 Comparison of QOM of motor activity log between groups ${ }^{*} P<0.05$, EG 1: Experimental group 1, EG 2: Experimental group 2, QOM: Quality of movement

\section{Discussion}

Stroke refers to damage to the nervous system caused by the necrosis of nerve cells in a brain area after the blood supply to the cranial nerve cells is blocked due to a blockage or rupture of a blood vessel in the brain. Such blood vessels supply oxygen to various areas of the brain [7].

Most stroke survivors develop sensory, motor, and cognitive impairments that limit their ability to perform basic daily living activities. Mainly, they experience serious impairments to their balance, motor ability, and daily living activities. Cerebrovascular diseases are a major cause of death, along with cancer and heart disease [8]. 
The frequency of stroke, which is more common among elderly patients, is increasing due to the current population's rising average age, but the rate of death due to stroke is decreasing - thanks to the development of medical technologies. Hemiplegic stroke patients experience problems in body alignment due to the paralysis of the muscles on their damaged sides. Moreover, the range of motion and coordination of their damaged upper limbs decrease due to indirect factors, such as increased muscle tone and repetitive abnormal movements, as well as direct reasons-such as innervation damage [9].

Additionally, a problem in processing and integrating sensory and movement information can occur due to a defect in the sensorimotor system and the loss of proprioception, leading to defects in position and movement control. Reportedly, due to such sensory losses, balance abilities decrease, such that patients experience a damaging fall at least once within six months of a stroke [10].

The purpose of upper extremity rehabilitation treatment for stroke patients is to improve the quality of life by minimizing the patient's disability and maximizing the patient's functions. As upper extremity rehabilitation treatment techniques for stroke patients, task-oriented therapy, forced-guided exercise therapy, mirror therapy, virtual reality therapy, and music therapy are being implemented in clinical practice. As representative neuromuscular stimulation treatments, proprioceptive neuromuscular stimulation and taping therapy are being applied in clinical practice [11-13].

In addition, therapeutic training using mirrors is an intervention method that can be easily applied in clinical practice and can help patients improve their motor function by exercising directly. As the effect of task-oriented therapy in stroke patients is well known, mirror therapy will also promote brain plasticity and recovery by performing direct, purposeful, and task-oriented movements of the arms and hands [14]. In this study as well, in the experimental group to which mirror therapy was applied, an increase in the patient's function and activities of daily living was observed in the posttest compared to the pre-test. This result is thought to be because motor learning occurred because immediate feedback was provided through mirror therapy.

Kinesio taping is an elastic taping method used to suppress muscle tension or promote activity by attaching it to the skin above the muscle to facilitate the movement of the prime mover. The key mechanism by which kinesio taping mediates muscle movement has not been clearly reported, but it has been suggested that it may improve lymph and blood circulation, reduce pain due to nerve inhibition, relieve abnormal muscle tension, and play an adjuvant role in joint repositioning. Since taping treatment has no side effects and is simple, it can be easily treated at home, but the biggest advantage is that it can be applied simultaneously with other treatments $[15,16]$.

In this study, an increase in upper extremity function and activities of daily living was observed in the group where taping was applied before proprioceptive neuromuscular facilitation treatment. This result is thought to be because taping stimulated proprioceptive sensation and increased the effect of proprioceptive neuromuscular facilitation.

\section{Conclusion}

This study was conducted to investigate the effect of mirror therapy and taping therapy, which can increase proprioceptive sensation and feedback stimulation, on upper extremity function and daily activities of stroke patients. In the results of this study, it was confirmed that the upper extremity function and daily activities of stroke patients were statistically increased by mirror therapy and taping therapy.

In other words, by applying mirror therapy and taping therapy that can increase proprioceptive sensation and feedback information in stroke patients, upper extremity function and daily activities can be increased. In this study, the number of subjects was small and quantitative evaluation was not possible. In the future, it is hoped that a quantitative evaluation method and a study applying mirror treatment and taping to various areas will proceed by supplementing the limitations of this study.

\section{Compliance with ethical standards}

\section{Acknowledgments}

This work was supported by the National Foundation of Korea (NRF) grant funded by the Korea government (MSIT) (No-2019R1F1A1057731). 


\section{Disclosure of conflict of interest}

No conflict of interest.

\section{Statement of informed consent}

Informed written consent were obtained from all individual participants included in the study.

\section{References}

[1] Proske U, Gandevia SC. The Proprioceptive Senses: Their Roles in Signaling Body Shape, Body Position and Movement, and Muscle Force. Physiol Rev. 2012; 92(4): 1651-1697.

[2] Tuthill JC, Azim E. Proprioception. Curr Biol CB. 2018; 28(5): R194-203.

[3] Hillier S, Immink M, Thewlis D. Assessing Proprioception: A Systematic Review of Possibilities. Neurorehabil Neural Repair. 2015; 29(10): 933-949.

[4] Ferris JK, Inglis JT, Madden KM, Boyd LA. Brain and Body: A Review of Central Nervous System Contributions to Movement Impairments in Diabetes. Diabetes. 2020; 69(1): 3-11.

[5] Taylor MD, Holdeman AS, Weltmer SG, Ryals JM, Wright DE. Modulation of Muscle Spindle Innervation by Neurotrophin-3 Following Nerve Injury. Exp Neurol. 2005; 191(1): 211-222.

[6] Defebvre L, Krystkowiak P. Movement Disorders and Stroke. Rev Neurol (Paris). 2016; 172(8-9): 483-487.

[7] Montaño A, Hanley DF, Hemphill JC. Hemorrhagic Stroke. Handb Clin Neurol. 2021; 176: 229-248.

[8] Raghavan P. Upper Limb Motor Impairment After Stroke. Phys Med Rehabil Clin N Am. 2015; 26(4): 599-610.

[9] Fong KNK, Ting KH, Chan CCH, Li LSW. Mirror Therapy with Bilateral Arm TTraining for Hemiplegic Upper Extremity Motor Functions in Patients with Chronic Stroke. Hong Kong Med J Xianggang Yi Xue Za Zhi. 2019; 3(1): $30-34$.

[10] Ikbali Afsar S, Mirzayev I, Umit Yemisci O, Cosar Saracgil SN. Virtual Reality in Upper Extremity Rehabilitation of Stroke Patients: A Randomized Controlled Trial. J Stroke Cerebrovasc Dis Off J Natl Stroke Assoc. 2018; 27(12): 3473-3478.

[11] Lisalde-Rodríguez ME, Garcia-Fernández JA. Mirror Therapy in Hemiplegic Patient. Rev Neurol. 2016; 62(1): 2836.

[12] Gunning E, Uszynski MK. Effectiveness of the Proprioceptive Neuromuscular Facilitation Method on Gait Parameters in Patients with Stroke: A Systematic Review. Arch Phys Med Rehabil. 2019; 100(5): 980-986.

[13] Huang YC, Leong CP, Wang L, Wang LY, Yang YC, Chuang CY, et al. Effect of Kinesiology Taping on Hemiplegic Shoulder Pain and Functional Outcomes in Subacute Stroke Patients: A Randomized Controlled Study. Eur J Phys Rehabil Med. 2016; 52(6): 774-781.

[14] Pérez-Cruzado D, Merchán-Baeza JA, González-Sánchez M, Cuesta-Vargas AI. Systematic Review of Mirror Therapy Compared with Conventional Rehabilitation in Upper Extremity Function in Stroke Survivors. Aust Occup Ther J. 2017; 64(2): 91-112.

[15] Huang YC, Chen PC, Tso HH, Yang YC, Ho TL, Leong CP. Effects of Kinesio Taping on Hemiplegic Hand in Patients with Upper Limb Post-Stroke Spasticity: A Randomized Controlled Pilot Study. Eur J Phys Rehabil Med. 2019; 55(5): 551-557.

[16] Hsieh HC, Liao RD, Yang TH, Leong CP, Tso HH, Wu JY, et al. The Clinical Effect of Kinesio Taping and Modified Constraint-Induced Movement Therapy on Upper Extremity Function and Spasticity in Patients with Stroke: A Randomized Controlled Pilot Study. Eur J Phys Rehabil Med. 2021; 57(4): 511-519. 